\title{
Proton pump inhibition induces autophagy as a survival mechanism following oxidative stress in human melanoma cells
}

\author{
ML Marino ${ }^{1}$, S Fais ${ }^{1}$, M Djavaheri-Mergny ${ }^{2,3}$, A Villa $^{4}$, S Meschini ${ }^{5}$, F Lozupone ${ }^{1}$, G Venturi ${ }^{1}$, P Della Mina ${ }^{4,6}$, S Pattingre ${ }^{7}$, \\ L Rivoltini ${ }^{6}, \mathrm{P}$ Codogno ${ }^{2}$ and A De Milito ${ }^{\star, 1,8}$
}

Proton pump inhibitors (PPI) target tumour acidic $\mathrm{pH}$ and have an antineoplastic effect in melanoma. The PPI esomeprazole (ESOM) kills melanoma cells through a caspase-dependent pathway involving cytosolic acidification and alkalinization of tumour pH. In this paper, we further investigated the mechanisms of ESOM-induced cell death in melanoma. ESOM rapidly induced accumulation of reactive oxygen species (ROS) through mitochondrial dysfunctions and involvement of NADPH oxidase. The ROS scavenger N-acetyl-L-cysteine (NAC) and inhibition of NADPH oxidase significantly reduced ESOM-induced cell death, consistent with inhibition of cytosolic acidification. Autophagy, a cellular catabolic pathway leading to lysosomal degradation and recycling of proteins and organelles, represents a defence mechanism in cancer cells under metabolic stress. ESOM induced the early accumulation of autophagosomes, at the same time reducing the autophagic flux, as observed by WB analysis of LC3-II accumulation and by fluorescence microscopy. Moreover, ESOM treatment decreased mammalian target of rapamycin signalling, as reduced phosphorylation of p70-S6K and 4-EBP1 was observed. Inhibition of autophagy by knockdown of Atg5 and Beclin-1 expression significantly increased ESOM cytotoxicity, suggesting a protective role for autophagy in ESOM-treated cells. The data presented suggest that autophagy represents an adaptive survival mechanism to overcome drug-induced cellular stress and cytotoxicity, including alteration of $\mathrm{pH}$ homeostasis mediated by proton pump inhibition.

Cell Death and Disease (2010) 1, e87; doi:10.1038/cddis.2010.67; published online 21 October 2010

Subject Category: Cancer

The biochemical and physical properties of the tumour microenvironment represent crucial factors that govern tumour progression and drive the selection of malignant cells. ${ }^{1}$ Tumour acidity is an inevitable consequence of the upregulated glycolytic flux induced by hypoxia, and dramatically affects cancer progression and sensitivity to therapy. ${ }^{2-4}$ An important feature of the tumour microenvironment is alteration of $\mathrm{pH}$ gradients the regulation of which is finely mediated by ion pumps such as vacuolar ATPase (V-ATPase), $\mathrm{Na}^{+} / \mathrm{H}^{+}$exchanger and carbonic anhydrases. Increased acid production following metabolic reprogramming of cancer cells results in acidification of extracellular $\mathrm{pH}(\mathrm{pHe})$ and alkalinization of intracellular/cytosolic $\mathrm{pH}(\mathrm{pHi}) .{ }^{5-8}$ Acidosis is indeed a hallmark of cancer and exerts a selective pressure driving malignant progression; it is also involved in metastatic potential, invasiveness and resistance to therapy. ${ }^{8-15}$ Thus, agents altering tumour $\mathrm{pH}$ homeostasis exert antitumour effects by inducing reversion of drug resistance and inhibiting tumour growth and metastatic progression. ${ }^{11,16-19}$

The V-ATPase is a proton pump translocating protons across membranes, thus acidifying the lumen of endolysosomal organelles and the extracellular environment in certain specialized cells. ${ }^{5}$ The role of V-ATPase in tumour progression is supported by its increased activity and expression in metastatic tumours. ${ }^{20}$ In fact, inhibition of V-ATPase reduces tumour growth and metastatic spread, thus representing a suitable therapeutic target. ${ }^{13,18}$ Several studies have shown that proton pump inhibitors (PPIs) such as omeprazole, esomeprazole (ESOM) and pantoprazole have an antineoplastic activity towards human hematopoietic and solid tumours. PPI revert chemoresistance in drug-resistant tumours and directly induce tumour cell killing. ${ }^{11,17,21,22}$ PPI treatment disturbs tumour $\mathrm{pH}$ gradients with major consequences on drug retention and traffic of acidic vesicles in human melanoma and gastric adenocarcinoma. ${ }^{17}$

\footnotetext{
${ }^{1}$ Department of Therapeutic Research and Medicines Evaluation, Unit of Antitumor Drugs, Istituto Superiore di Sanità, Rome, Italy; ${ }^{2}$ Université Paris-Sud, INSERM U756, Châtenay-Malabry, France; ${ }^{3}$ INSERM U916, VINCO Institut Bergonié 229, cours de l'Argonne, Bordeaux Cedex 33076, France; ${ }^{4}$ University of Milano-Bicocca, Microscopy and Image Analysis Consortium, Monza, Italy; ${ }^{5}$ Department of Technology and Health, Istituto Superiore di Sanità, Rome, Italy; ${ }^{6}$ Fondazione IRCCS Istituto Nazionale Tumori, Unit of Immunotherapy of Human Tumours, Milan, Italy; ${ }^{7} \mathrm{IRCM}$, Institut de Recherche en Cancérologie de Montpellier, CRLC Val d'Aurelle-Paul Lamarque, Montpellier, France and ${ }^{8}$ Department of Oncology-Pathology, Cancer Center Karolinska, Karolinska Institute, Stockholm, Sweden

${ }^{*}$ Corresponding author: A De Milito, Department of Therapeutic Research and Medicines Evaluation, Unit of Antitumor Drugs, Istituto Superiore di Sanità, Viale Regina Elena 299, 00161, Rome, Italy. Tel: + 39064990 2153; Fax: + 39064990 3691; E-mail: angelo.demilito @ iss.it or angelo.de-milito@ ki.se Keywords: proton pumps; tumour pH; autophagy; ESOM; V-ATPase

Abbreviations: PPI, proton pump inhibitors; ESOM, esomeprazole; ROS, reactive oxygen species; V-ATPase, vacuolar ATPase; pHi, intracellular pH; NAC, N-acetylL-cysteine; DPI, diphenylene-iodonium; DHR123, dihydrorhodamine123; HE, dihydroethidium; siRNA, small interfering RNAs; Baf-A1, bafilomycin A1

Received 01.6.10; revised 17.8.10; accepted 16.9.10; Edited by D Bano
} 
Recently, we reported that PPI have a caspase- and $\mathrm{pH}$-dependent antineoplastic activity in human melanoma and that treatment of human melanoma xenografts with PPI reduces tumour growth through a dramatic disturbance of tumour $\mathrm{pH}$ homeostasis. ${ }^{11}$

Autophagy is a tightly regulated catabolic mechanism used by eukaryotic cells to degrade long-lived proteins and organelles. $^{23}$ The autophagic pathway starts with the formation of isolation membranes that elongate to form the autophagosome, a double-membrane vesicle that sequesters cytoplasmic material. After fusion of autophagosomes with lysosomes, the autophagic cargo is degraded and recycled back to the cytoplasm. ${ }^{24}$ Acidic organelles such as lysosomes and autophagic vacuoles may have a key role in tumour malignancy, and modulation of autophagy has been proposed as possible anticancer strategy. However, autophagy may have both tumour-suppressing and tumour-promoting roles, making it complex to identify strategies to target autophagy in cancer therapy. ${ }^{25}$ We previously showed that PPI cause alkalinization of lysosomal $\mathrm{pH}$ and induce reactive oxygen species (ROS) in B-cell tumours; ${ }^{17,22}$ both these events are known to modulate autophagy.
In this study, we used human melanoma cell lines to identify the upstream mechanisms mediating the antineoplastic effect of ESOM. We found that ESOM induced a ROS-dependent cell death and that autophagy represents an adaptive response to oxidative stress induced by ESOM in human melanoma cells.

\section{Results}

ESOM induces ROS-dependent cell death in human melanoma. We reported that targeting tumour acidic $\mathrm{pH}$ by ESOM induced cell death in human melanoma through a caspase-dependent pathway and reduced tumour growth in SCID mice engrafted with human melanoma. ${ }^{22}$ ESOM cytotoxic effects were detected in unbuffered culture conditions and dramatically enhanced in acidic culture conditions. Here, we evaluated which upstream events triggered ESOM-induced cell death in human melanoma. Treatment with ESOM rapidly induced accumulation of hydrogen peroxide and superoxide radicals in melanoma cells, as shown by DHR123 and HE fluorescence, respectively (Figure 1a). a
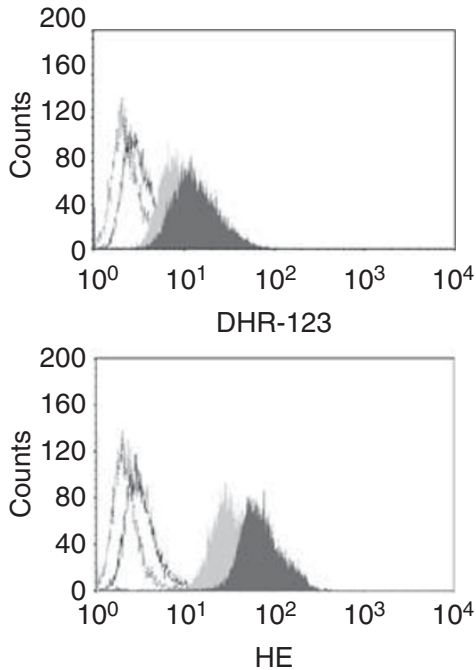

C

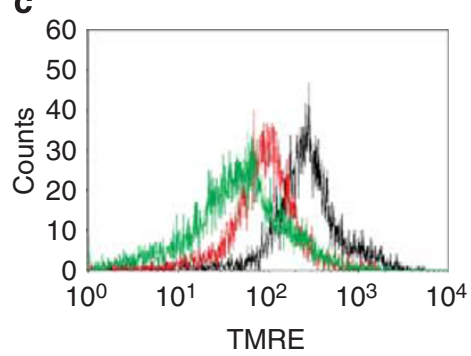

b

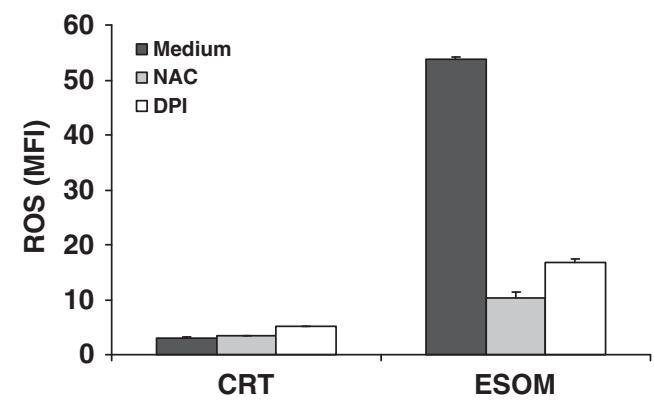

d

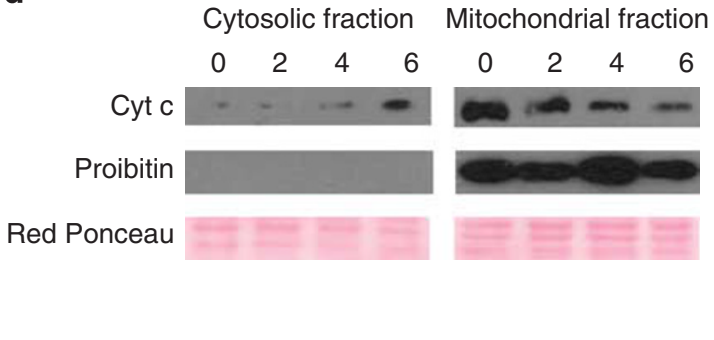

Figure 1 ESOM induces ROS and mitochondrial alterations in human melanoma. (a) ESOM (160 $\mu \mathrm{M})$ induces accumulation of hydrogen peroxide and superoxide radicals, as shown by DHR123 and HE fluorescence, respectively. In the histogram, untreated Me30966 cells are indicated by the dotted line and cells treated with ESOM after 1,2 and $4 \mathrm{~h}$ are indicated with black line, light grey and dark grey histograms, respectively. (b) ESOM-induced ROS accumulation is inhibited by both the ROS scavenger NAC and the NADPH oxidase inhibitor DPI in Me30966 cells. (c) Me30966 cells treated with ESOM (160 $\mu \mathrm{M})$ for $5 \mathrm{~h}$ show mitochondrial membrane depolarization detected by TMRE probe. Black, red and green lines indicate untreated, ESOM-treated and staurosporine-treated cells, respectively. (d) Me30966 cells treated with ESOM (160 $\mu \mathrm{M}$ ) for 2, 4 and $6 \mathrm{~h}$ were processed to purify the cytosolic and mitochondrial fractions. Expression of cytochrome $c$ was assessed by WB and indicates a time-dependent release of the protein from the mitochondria into the cytosol. The Red Ponceau staining indicates equal loading of proteins, whereas purity of the fractions was assessed by proibitin expression 
The major sources of intracellular ROS are mitochondria and NADPH oxidase and we analysed their possible involvement in driving ESOM-mediated ROS accumulation. First, we observed that diphenylene-iodonium (DPI), a specific pharmacological inhibitor of NADPH oxidase, and the common ROS scavenger $N$-acetyl-L-cysteine (NAC) significantly reduced ROS accumulation in cells treated with ESOM (Figure 1b). Moreover, TMRE staining after ESOM treatment showed depolarization of mitochondrial membrane (Figure 1c), whereas release of cytochrome $c$ from mitochondria and its accumulation in the cytosolic fraction were observed by western blot in a time-dependent manner and within $6 \mathrm{~h}$ after treatment (Figure 1d). These experiments indicate that both mitochondria and NADPH oxidase participate in ROS induction by ESOM in human melanoma cells.

We evaluated the involvement of ROS in ESOM-induced apoptosis in Me30966 and Mel501. These cells pretreated with NAC $(5 \mathrm{mM})$ were completely protected from cell death induced by $100 \mathrm{mM} \mathrm{H}_{2} \mathrm{O}_{2}$ (data not shown). As shown in the morphological analysis of Me30966 cells (Figure 2a), pretreatment with NAC completely abrogated ESOM-induced cell death in both Mel501 and Me30966 cells (Figure 2b). As we reported that ESOM-induced apoptosis is caspase dependent, we observed here that the inhibition of apoptosis in the presence of NAC was associated with decreased percentage of cells with active caspase-3 and caspase-8 (Figure 2c). We also observed that inhibition of NADPH oxidase by DPI induced a significant reduction of ESOM-induced cell death in Me30966 cells (Supplementary Figure 1a).
$\mathrm{PPI}$ are known to induce cytosolic acidification in cancer cells ${ }^{11,22}$ and we found that ESOM-induced acidification of cytosolic $\mathrm{pH}$ was significantly prevented by NAC in Me30966 cells (Figure 2d, $P<0.01$ ), suggesting that ROS production is an early event upstream of ESOM effects on cytosolic $\mathrm{pH}$ and alteration of intracellular $\mathrm{pH}$ gradients.

ESOM induces the accumulation of autophagic vacuoles in melanoma cells. It is known that ROS are involved in the autophagic response of cancer cells to nutrient starvation or metabolic stress. ${ }^{26}$ Moreover, PPIs have been reported to cause alkalinization of lysosomal $\mathrm{pH}$ (Supplementary Figure 1b) and disturbance of acidic vesicle traffic. As proper lysosomal function and ROS are crucial autophagy regulators, we investigated whether ESOM-induced cytotoxicity involved alteration in the autophagic pathway. ESOM-treated cells showed massive vacuolization (Figure 3a, upper panel), which has been reported to occur in cells before apoptosis. ${ }^{27}$ Electron microscopy experiments showed that treatment of Me30966 with ESOM leads to an increased number of autophagic vacuoles per cell as compared with untreated cells (Figure $3 a$, middle and lower panels). To evaluate autophagy, we looked at the cellular distribution of the microtubule-associated protein 1 light chain 3 (LC3), a marker of autophagy. In conditions of autophagy activation, cytosolic LC3 is conjugated to phosphatidylethanolamine (LC3-II) and localizes to autophagosomes. Therefore, the number of LC3-positive vesicles a
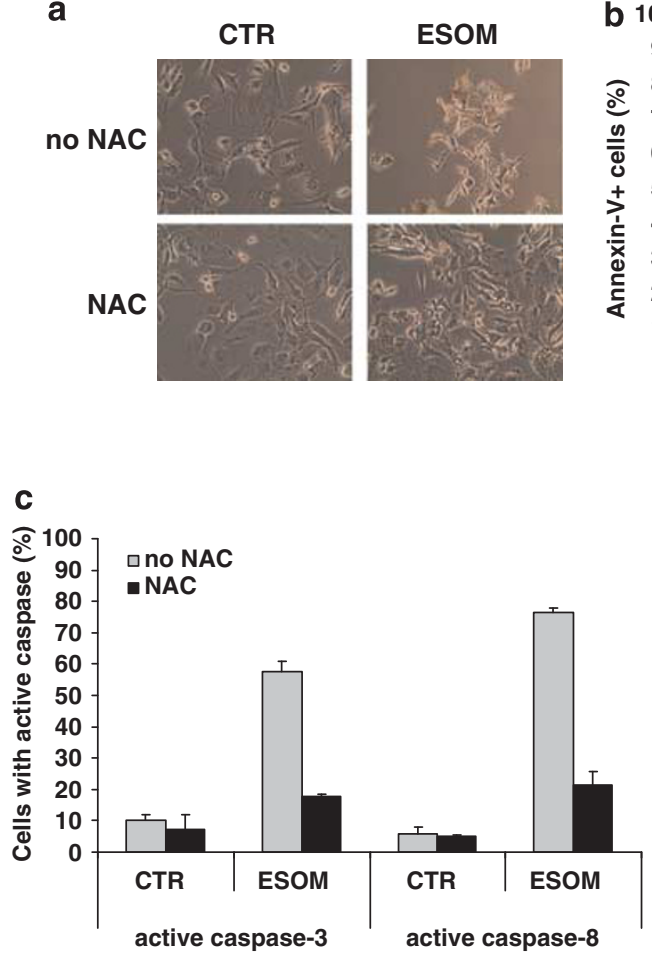

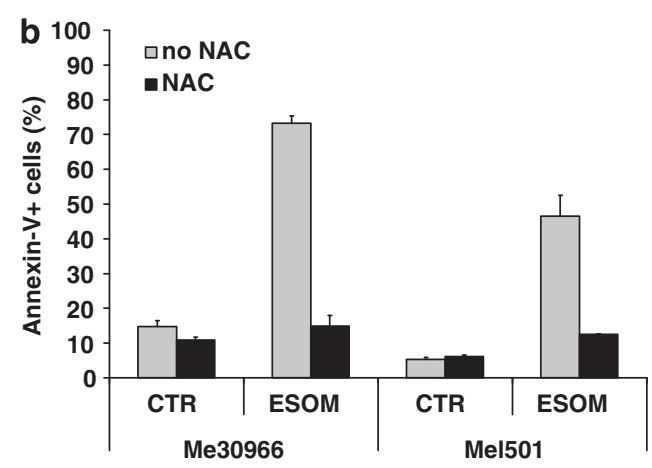

d

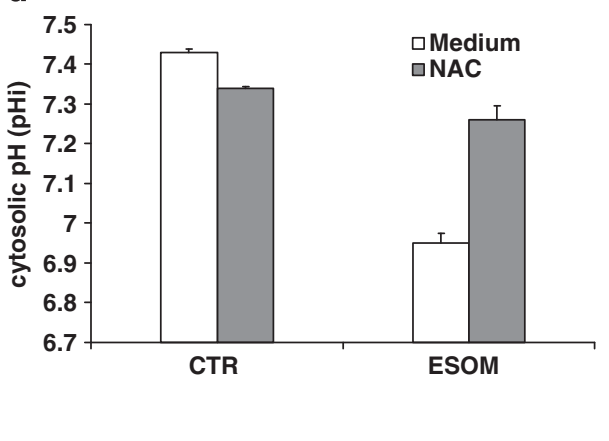

Figure 2 Involvement of ROS in ESOM-induced cell death. (a) The presence of NAC protects Me30966 cells from ESOM-induced cell death as observed by light microscopy. (b and c) ROS scavenging by NAC inhibits ESOM-induced apoptosis and caspase activation in both Me30966 and Mel501 cells. (d) ESOM-induced cytosolic acidification is inhibited by NAC in Me30966 cells treated with ESOM for $6 \mathrm{~h}$. In all these experiments, $160 \mu \mathrm{M}$ ESOM was used 
a

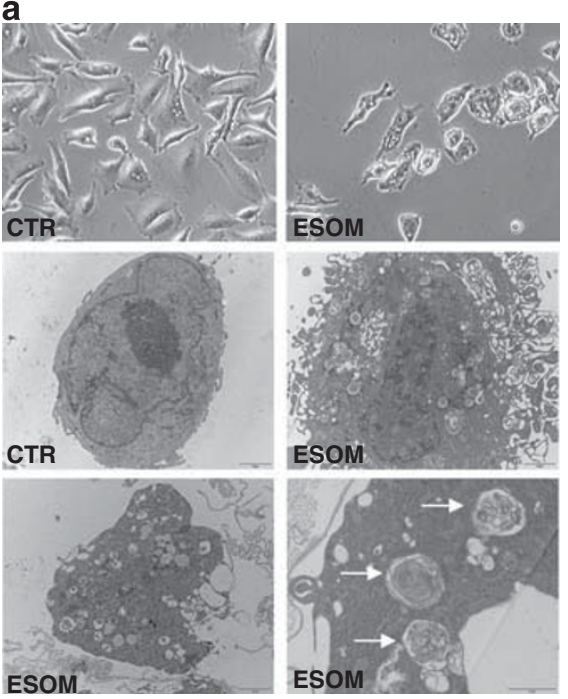

b
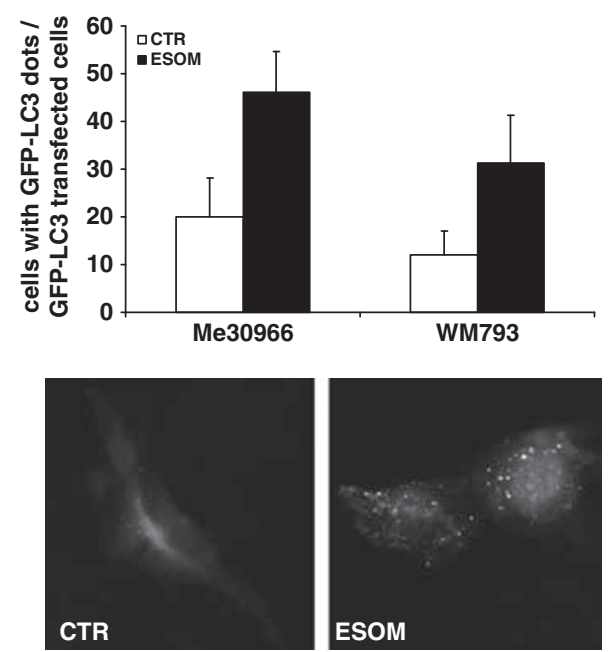

d

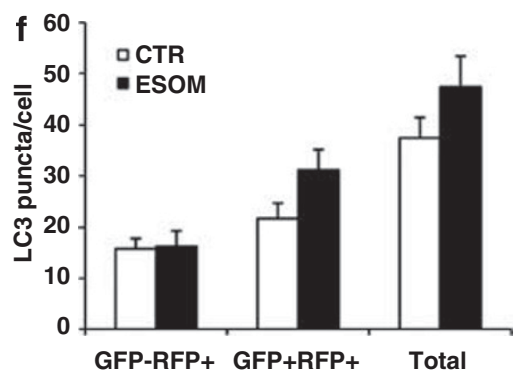

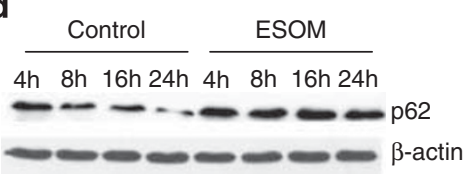
$4 \mathrm{~h} 8 \mathrm{~h} 16 \mathrm{~h} 24 \mathrm{~h} 4 \mathrm{~h} 8 \mathrm{~h} 16 \mathrm{~h} 24 \mathrm{~h}$ $-\cdots-{ }_{-1-a c t i n}$ GFP-RFP+ GFP+RFP+ Total e
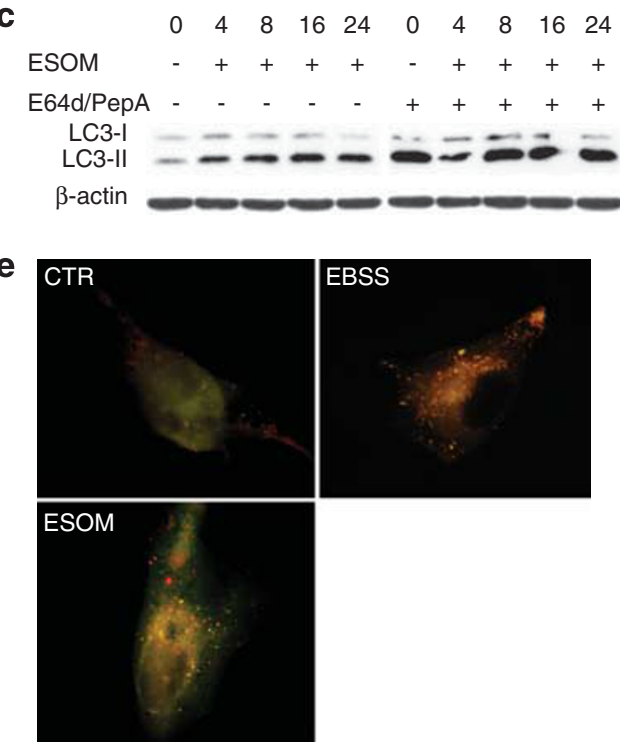

Figure 3 Effects of ESOM on autophagic flux. (a) Me30966 cells treated with ESOM (160 $\mu \mathrm{M})$ for $8 \mathrm{~h}$ are characterized by massive intracellular vacuolization. (b) Treatment with ESOM (for $8 \mathrm{~h}$ ) induces accumulation of LC3-positive autophagic vacuoles in Me30966 and WM793 cells. (c) ESOM induces accumulation of the LC3-II form in autophagy-competent melanoma cells, as shown by the further accumulation of LC3-II in cells pretreated with inhibitors of lysosomal proteases. (d) ESOM-induced alteration of the autophagic flux is confirmed by accumulation of p62. (e and $\mathbf{f}$ ) Analysis of the mRFP-GFP-LC3-plasmid shows the accumulation of autophagosomes in ESOMtreated Me30966 cells. Cells cultured in EBSS were used as positive control

and the amount of LC3-II are commonly used as a measure of autophagosome formation. ${ }^{28}$ Following cell transfection with GFP-LC3-expressing plasmid, ESOM strongly enhanced the appearance of punctuate fluorescence in Me30966 (Figure 3b, lower panel) and WM793 cells, indicating a redistribution of LC3 to autophagic vacuoles with respect to untreated cells. Quantitative evaluation of cells carrying GFP-LC3-positive dots showed that ESOM treatment significantly increased the percentage of cells carrying autophagosome markers (Figure 3b, upper panel). Similar results were observed on Me30966 cells when endogenous LC3 fluorescence pattern was analysed (Supplementary Figure 1c).
ESOM induces autophagosome accumulation and slows down the autophagic flux. Increased autophagosome markers may be explained by increased autophagosome formation due to induction of autophagic activity, or accumulation of autophagic organelles due to impaired degradation of autolysosome content. Autophagic flux may be measured by the levels of LC3-II degraded in autolysosomes in which lysosomal hydrolases are functional. $^{28}$ Thus, the status of autophagic flux in Me30966 cells was analysed in the presence or absence of lysosomal protease inhibitors (E64d/Pepstatin A). First, we observed that these cells are fully competent for starvation-induced autophagy (Supplementary Figure 1d). 
We observed that ESOM induced a time-dependent accumulation of LC3-II in Me30966 cells (Figure 3c), which was further enhanced in the presence of E64d/PepA, supporting the notion that, in cells treated with ESOM, the autophagic flux is not blocked. However, the effect of lysosomal inhibitors on LC3-II accumulation in control cells is increased with respect to ESOM-treated cells, also indicating that ESOM partially reduces the autophagic flux (Supplementary Figure 1e). These findings suggest that ESOM likely causes the accumulation of autophagosomes and slows down the autophagic flux. In line with the accumulation of LC3-II, Me30966 cells treated with ESOM showed accumulation of p62 detected as early as $4 \mathrm{~h}$ after treatment (Figure 3d).

These data were further confirmed by the analysis of cells transfected with an mRFP-GFP-LC3-expressing plasmid. This probe allows distinguishing between autophagosomes (GFP + RFP + yellow puncta) and autolysosomes (GFPRFP + red puncta), as the GFP fluorescence is quenched in the acidic lumen of autolysosomes. ${ }^{28}$ In fact, ESOM-treated cells carried an increased number of dots with RFP + GFP + fluorescence with respect to untreated cells (Figure $3 e$ and $f$ ), whereas the number of RFP + GFP - dots was not affected, suggesting that ESOM induces the accumulation of autophagosomes as it slows down the autophagic flux.

As ESOM-induced cell death was mediated by massive ROS production, we evaluated the effects of ROS inhibition on ESOM-induced alterations in the autophagic flux. The expression and localization of endogenous LC3 in Me30966 cells was studied by laser scanning confocal microscopy (LSCM) analysis. Basal levels of LC3-positive autophagic vesicles were significantly increased $8 \mathrm{~h}$ after ESOM treatment, with a significant proportion of cells showing a clear punctuate fluorescence (Figure $4 a$ and b). The presence of lysosomal protease inhibitors did not significantly affect ESOM-induced accumulation of endogenous LC3 dots, confirming that ESOM partially reduces the autophagic flux (Figure $4 \mathrm{a}$ and b). Conversely, pretreatment with NAC prevented the accumulation of LC3-positive dots induced by ESOM (Figure 4a and b) and inhibited LC3-II (Figure 4c), suggesting that ROS are involved in ESOM-induced accumulation of autophagosomes in melanoma cells.

ESOM inhibits the mammalian target of rapamycin (mTOR)-signalling pathway. An important pathway regulating autophagy is the signalling through the mTOR, the inhibition of which activates autophagy in cells under nutrient starvation. ${ }^{29}$ Decreased mTOR signalling can be evaluated by the reduced phosphorylation of $\mathrm{p} 70-\mathrm{S} 6 \mathrm{~K}$ and 4EBP1, which are two important downstream targets of mTOR. We observed that, in Me30966 cells treated with ESOM, the phosphorylation of both p70-S6K and 4EBP1 was decreased in a time-dependent manner and with similar time kinetics (Figure 4d). In line with the role of ROS in driving autophagy, we observed that the inhibition of mTOR activity was prevented by ROS scavenging, as the presence of NAC partially restored the phosphorylation of both 4EBP1 and p70 (Figure 4e). These findings further confirm that ESOM induces the accumulation of autophagosomes and suggests that inhibition of the mTOR signalling pathway may drive the autophagic response induced by ESOM in melanoma cells.

Inhibition of autophagy increased PPI cytotoxicity in melanoma cells. To analyse the role of autophagy in ESOM-induced cell death, we measured cell death in Me30966 and WM793 cells in which autophagy was inhibited by knockdown of Beclin-1 or Atg-5 gene expression or by inhibition of autolysosome formation by Baf-A1. First, we observed that pretreatment of melanoma cells Me30966 with Baf-A1 significantly increased the cytotoxicity of ESOM in melanoma cells (Figure 5a). In line with this result, we observed that, in ESOM-treated Me30966 cells, there is an increased accumulation of LC3-II in the presence of Baf-A1. However, the effect of Baf-A1 in ESOM-treated cells was reduced when compared with control cells, further confirming that ESOM also slows down the autophagic flux (Figure 5a). We then measured ESOM-induced cell death in Me30966 and WM793 cells, in which the autophagic genes Atg5 and Beclin-1 were silenced. Knockdown of Atg5 and Beclin-1 decreased protein expression (Supplementary Figure 2A) and inhibited the formation of LC3 + puncta in GFP-LC3-transfected cells, as shown for Me30966 cells (Figure 5b).

Interestingly, knockdown of Atg5 (and to a lesser extent Beclin-1) increased ESOM-induced cell death in both cell lines (Figure 5c and d) and also in Mel501 cells (not shown), strongly indicating that autophagy (and autophagosome formation) may represent an adaptive survival mechanism used by melanoma cells in response to ESOM insult.

\section{Discussion}

The acidic $\mathrm{pH}$ of solid tumour tissues has been proposed as a therapeutic target and a drug delivery system for selective anticancer treatments. ${ }^{6,8,13,30,31}$ Inhibition of several proton extrusion mechanisms adopted by malignant cancer cells represents one promising therapeutic strategy ${ }^{6-8}$ and PPI treatment has been proposed as a valid and feasible approach because of the relatively low toxicity and potential selectivity of these drugs..$^{8,11,32}$ The antitumour activities of PPI include both, their ability to revert chemoresistance of drug-resistant tumours and their capacity to induce tumour cell killing. ${ }^{11,21,22}$ Preclinical studies reported recently by our group ${ }^{11,17,22}$ have provided the ground for currently ongoing phase II clinical trials evaluating ESOM, both in combination therapy and as a first-line treatment of metastatic melanoma, breast carcinoma and osteosarcoma in Italy and China. Interestingly, the pharmacological and chemical properties of PPI make these acid-activated prodrugs as ideal drugs for selective delivery at the acidic tumour site, where, once activated, they may exert their antineoplastic activity. ${ }^{11}$ In line with the feasibility of such an approach, a chemically modified omeprazole containing a NAC molecule (NACO) to increase bioavailability has been reported to induce apoptosis in human melanoma. ${ }^{33}$ Recently, we have reported in a preclinical model of human melanoma that ESOM treatment exerts a $\mathrm{pH}$-dependent antineoplastic activity through its ability to severely disturb tumour $\mathrm{pH}$ homeostasis. ${ }^{11}$ The proapoptotic effects of ESOM were mediated by the induction 

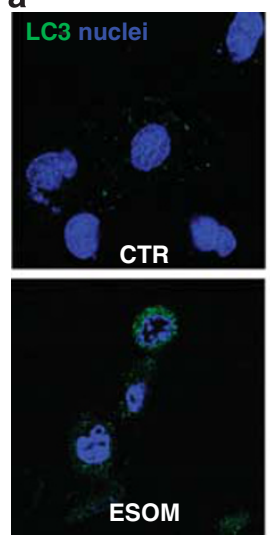

C b
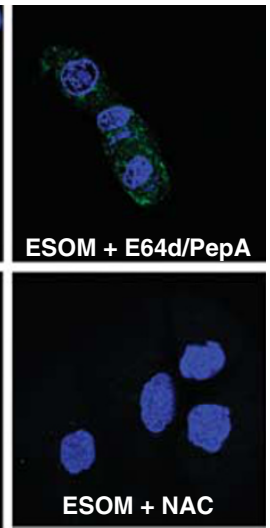

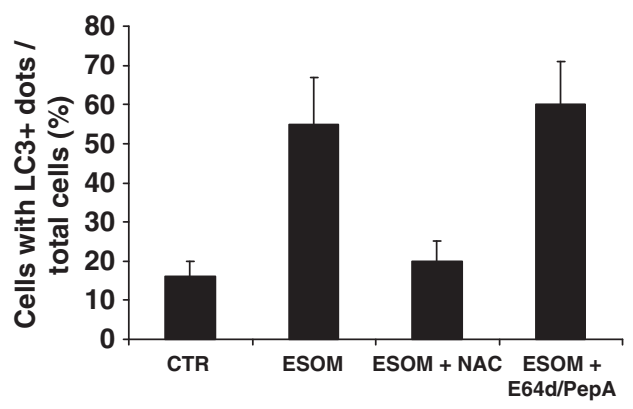

d

p-4EBP1

4EBP1

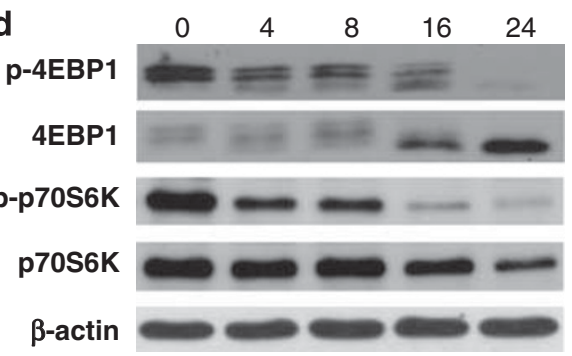

p70s6K

$\beta$-actin

e

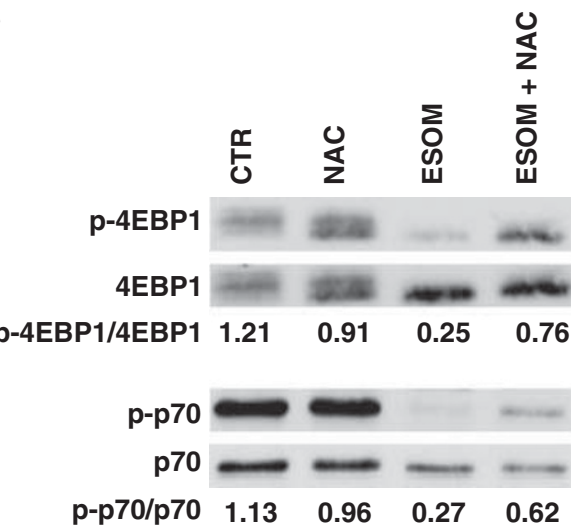

Figure 4 Role of ROS and mTOR in ESOM effects on the autophagic flux. (a and $\mathbf{b}$ ) NAC pretreatment prevents the accumulation of autophagosomes induced by ESOM $(160 \mu \mathrm{M})$ in Me30966 cells, as shown by confocal fluorescence analysis of endogenous LC3 and quantification of cells with LC3 + dots. (c) Western blot analysis of LC3-I and LC3-II in the presence or absence of NAC in ESOM-treated cells. (d and e) ESOM treatment inhibits the mTOR pathway as shown by the time-dependent reduced phosphorylation of 4-EBP1 and p70S6K proteins. Such inhibition is prevented by the ROS scavenger NAC

of a caspase-dependent pathway and acidification of cytosolic $\mathrm{pH}$. The aim of the current study was to further dissect the molecular and biochemical pathways activated in human melanoma cells treated with PPI (summarized in Figure 6). PPIs have been previously shown to induce caspaseindependent and partially ROS-dependent cell death in human B-cell tumours. ${ }^{22}$ Conversely, PPIs induce a caspase-dependent cell death in human melanoma ${ }^{11}$ and we reported here that accumulation of ROS is instrumental for ESOM-mediated cell death in human melanoma. Both mitochondrial and NADPH oxidase seemed to be involved in triggering ROS accumulation in ESOM-treated cells. Besides their function in triggering apoptosis, ROS may function as important signalling molecules in starvation-induced autophagy, ${ }^{26}$ and it was recently shown that ROS production induced by NADPH oxidase activates antibacterial autophagy in phagocytes, ${ }^{34}$ suggesting a role for NADPH oxidase in autophagy regulation. Indeed, we found a time-dependent induction of autophagy in ESOM-treated human melanoma cells, and inhibition of ROS by a ROS scavenger completely inhibited both apoptosis and autophagosome accumulation.

Targeting the autophagy regulation of cancer cells is a therapeutic strategy yet to be properly designed. In fact, the role of autophagy in cancer development, malignant progression and therapy is still under investigation. ${ }^{25,29}$ Despite the initial observation that the ATG gene Beclin-1 functioned as a tumour suppressor, ${ }^{35}$ several studies have suggested that autophagy may also function as a survival mechanism adopted by cancer cells facing hostile microenvironment conditions (such as hypoxia) and the resultant metabolic 


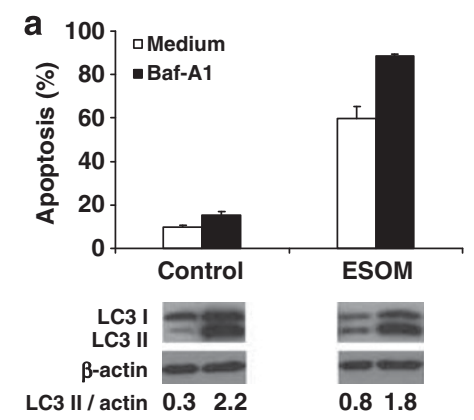

b
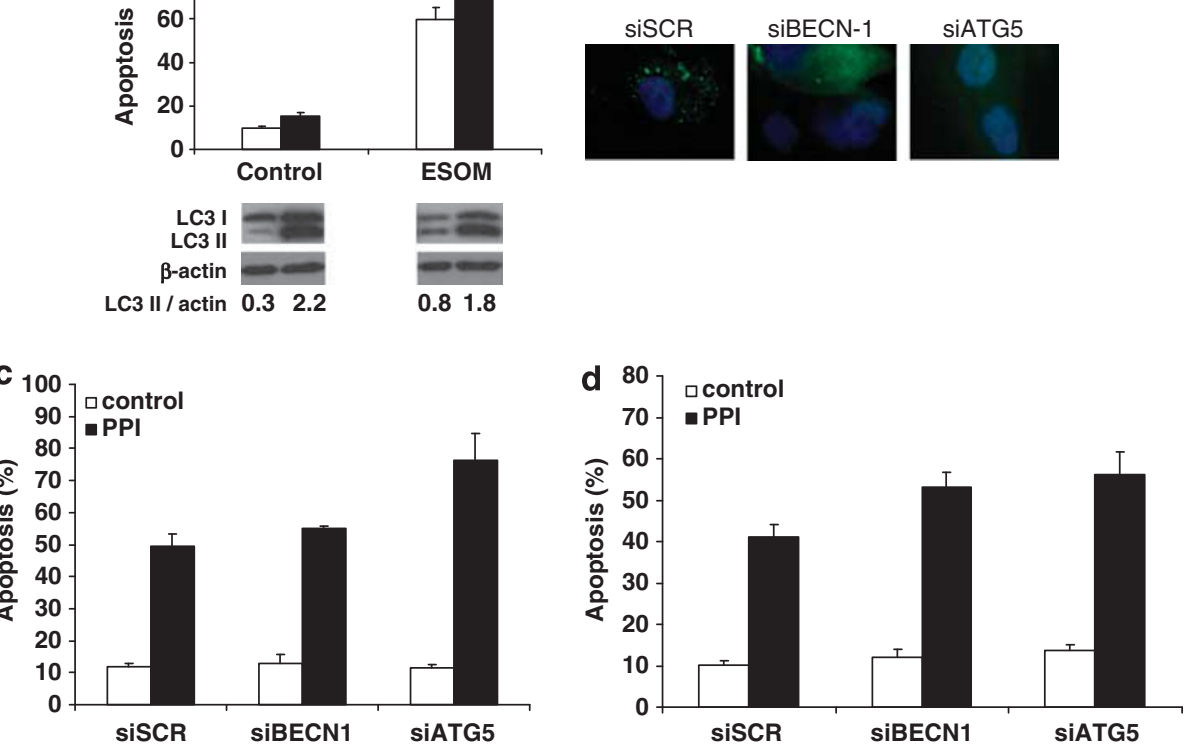

Figure 5 Autophagy is a cytoprotective mechanism in ESOM-treated melanoma cells. (a) Me30966 cells pretreated with Baf-A1 (40 nM) show increased sensitivity to ESOM-induced apoptosis in association with a further increased LC3-II accumulation. (b) Knockdown of Atg5 and beclin-1 induces a cytosolic localization of LC3 as compared with autophagosomal localization in control Me30966 cells. (c and d) Knockdown of Atg5 and beclin-1 increases the cytotoxicity of ESOM in Me30966 cells and WM793 cells, respectively

stress. Moreover, many currently used anticancer agents are able to induce the accumulation of autophagosomes in vitro, which likely represents a protective mechanism against the stress induced by cytotoxic agents. ${ }^{23-25}$ It has been recently reported that autophagy is activated in tumour cells that survive apoptosis following therapeutic stress. ${ }^{36}$ In this model, cloroquine treatment inhibited autophagy-dependent survival of tumour cells, indicating that autophagy may contribute to the selection of cells surviving chemotherapy. Interestingly, cloroquine is a lysosomotropic agent that raises lysosomal pH and induces accumulation of autophagosomes. ${ }^{37}$ Similarly, siramesine was reported as a lysosomotropic compound able to induce cytoprotective autophagosome accumulation in breast carcinoma cells. ${ }^{38}$ Interestingly, several PPIs (omeprazole, ESOM and pantoprazole) raise lysosomal $\mathrm{pH}$ and decrease intracellular $\mathrm{pH}$, a feature associated with their ability to sensitize cancer cells to chemotherapeutics as well. We found that in human melanoma cells in which autophagy was downregulated through gene silencing, the proapoptotic effects of ESOM were significantly enhanced, supporting evidence that an intact autophagic pathway is necessary to overcome ESOM cytotoxicity and that induction of autophagy in these cells represents an adaptive survival mechanism to ESOM-induced damage. In agreement with this, the analysis of cells collected at the end of a clonogenic survival assay in three melanoma cells lines strikingly indicated induction of autophagy (detected as LC3-II accumulation) in colonies surviving ESOM treatment (Supplementary Figure $2 \mathrm{C}$ and $\mathrm{D}$ ). Recently, the polyphenol kaempferol has been reported to trigger a ROS-dependent and caspase-dependent cell death in HeLa cells and these cells activate autophagy as a survival mechanism to the drug-induced bioenergetics failure. ${ }^{39}$
Moreover, desmethylclomipramine (the active metabolite of a drug used for the treatment of psychiatric disorders) was found to increase autophagosomal markers and block the degradation of autophagic cargo in HeLa cells. ${ }^{40}$ Treatment with desmethylclomipramine potentiated the cytotoxic effects of doxorubicin on HeLa cells through inhibition of the autophagic pathway. In light of the reported ability of PPI to revert chemoresistance of tumour cells, ${ }^{19,26,29}$ it is conceivable that this effect may be mediated by the inhibition of autophagy that we described here.

In conclusion, we report that treatment with ESOM induces a ROS-dependent cell death in human melanoma and that these cells use the autophagic pathway as a defence and adaptive mechanism. The findings reported in this study provide the rationale for possible combination therapies including PPI and other autophagy inhibitors for the treatment of melanoma.

\section{Materials and Methods}

Reagents. ESOM sodium salt (AstraZeneca, Mölndal, Sweden) was resuspended $2 \mathrm{mg} / \mathrm{ml}$ in $0.9 \% \mathrm{NaCl}$ (saline) immediately before use. Bafilomycin A1 (Baf-A1), hydrogen peroxide, NAC, DPI, E64d and phosphate-buffered saline (PBS) were from Sigma (Milano, Italy). RPMl 1640, antibiotics and fetal calf serum (FCS) were from Cambrex (Milano, Italy). Pepstatin A and Fugene were from Roche Applied Science (Milano, Italy). Dihydrorhodamine 123 (DHR123), dihydroethidium $(\mathrm{HE})$ and lipofectamine 2000 were from Invitrogen (Milano, Italy). The following antibodies were used: p70S6K, phospho-p70S6K, 4EBP1 and phospho-4EBP1 (Cell Signaling Technology, Milano, Italy), p62 (BD Transduction Laboratories, Milano, Italy), Beclin-1 and Atg5 (Santa Cruz Biotechnology, USA), $\beta$-actin (Cell Signaling Technology), antirabbit and antimouse-HRP-conjugated antibodies (Amersham Biosciences, Milano, Italy). Anti-LC3 antibody against a synthetic LC3 peptide was produced in rabbits by NeoMPS (Strasbourg, France). The GFPLC3 plasmid and the mRFP-GFP-LC3 plasmid were kind gifts from Dr. Tamotsu Yoshimori (National Institute of Genetics, Mishima, Japan). 


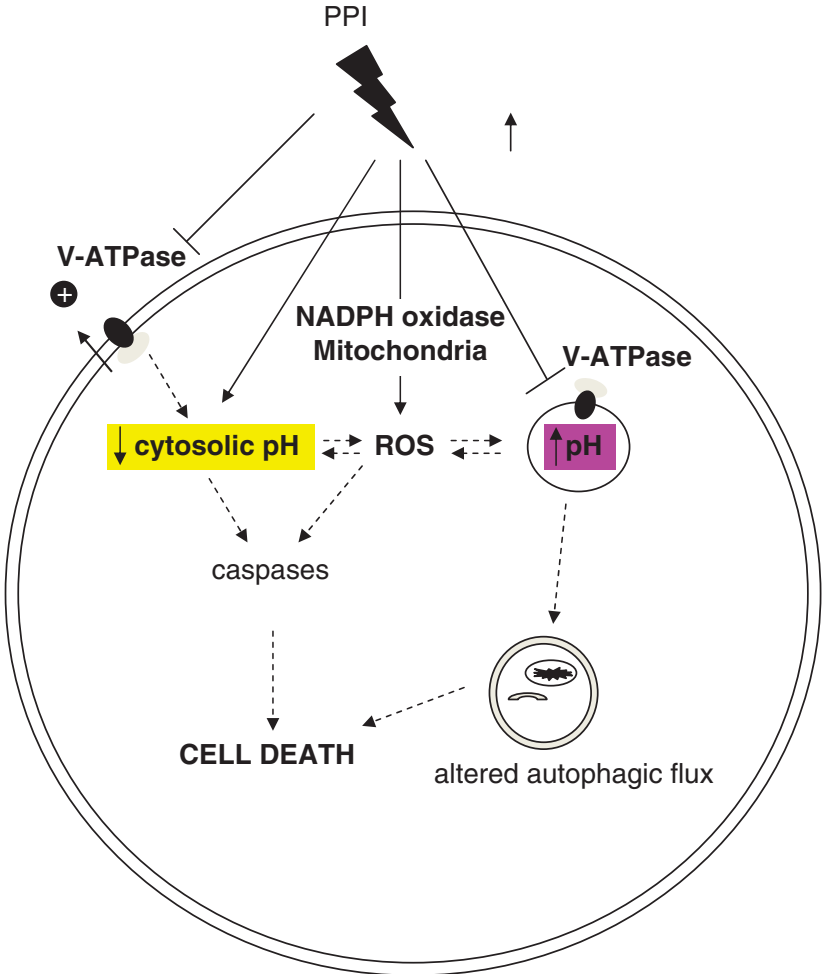

Figure 6 Schematic model of ESOM cytotoxic effects on melanoma cells. The picture shows the possible cellular pathways through which ESOM induces cell death in melanoma cells. Inhibition of proton extrusion is a crucial step in the antitumour activity of ESOM, as this effect causes acidification of the cytosol and alkalinization of lysosomes. The severe disturbance of cellular $\mathrm{pH}$ gradients and the accumulation of ROS, likely through the contribution of both NADPH oxidase and mitochondrial dysfunction, induce the activation of caspases and the alteration of autophagic flux, thus leading and contributing to cell death

Cell culture. Human melanoma cell lines Me30966, Mel501 and WM793 (a gift from Dr. Meenhard Herlyn, Wistar Institute, PA, USA) were cultured with RPMI 1640 in the presence of $10 \%$ FCS and antibiotics. Experiments were performed in unbuffered medium (without sodium bicarbonate).

Cell death determination. Cells were seeded at $15000-40000$ cells per well in 6-or 12-well plates in duplicate wells. The next day, ESOM was added at indicated concentrations and $24 \mathrm{~h}$ later cells were collected for analysis. Determination of cell death was performed by annexin-V-FITC and propidium iodide staining, following the manufacturer's instructions (Alexis Biochemicals, Firenze, Italy). Cells showing positivity for annexin-V binding were defined as apoptotic. Cells were sorted on a Becton Dickinson FACScan machine and analysed using CellQuest software (Becton Dickinson Systems, Milano, Italy). At least 10000 cells per sample were acquired.

Analysis of oxidative stress. Detection of ROS was evaluated on $0.25 \times 10^{6}$ cells at different time points after ESOM treatment. Hydrogen peroxide $(100 \mathrm{mM})$ was used as positive control for induction of ROS. HE and DHR123 were used to detect superoxide radicals and hydrogen peroxide, respectively. After treatment, cells were incubated with $10 \mu \mathrm{M} \mathrm{HE}$ or DHR123 in FCS-free RPMI for $10 \mathrm{~min}$ at $37^{\circ} \mathrm{C}$ and immediately analysed by FACS, collecting the fluorescence into FL3 and FL1 channels, respectively.

Western blot. Cells were collected and treated with RIPA buffer $(150 \mathrm{mM} \mathrm{NaCl}$, $50 \mathrm{mM}$ Tris with $\mathrm{pH} 7.4,1 \% \mathrm{NP} 40,0.1 \%$ SDS, $0.5 \%$ sodium deoxycholate) supplemented with protease and phosphatase inhibitors, and protein concentration of cellular extracts was determined by Bio-Rad Protein Assay (Bio-Rad Laboratories, Hercules, CA, USA). SDS-PAGE was performed on acrylamide gels (12-15\%) loading $30 \mu \mathrm{g}$ of proteins per lane. After electrophoresis, proteins were transferred to PVDF membranes and incubated for $2 \mathrm{~h}$ at RT in PBS with $5 \%$ non-fat dry milk.
Protein loading was assessed by Red Ponceau staining of the membranes. Membranes were incubated with primary antibodies overnight at $4{ }^{\circ} \mathrm{C}$, followed by appropriate HRP-conjugated secondary antibodies. Antibody staining was visualized by the ECL system (Pierce, Rockford, IL, USA).

LSCM analysis. Cell culture monolayers were flattened over a glass slide, fixed with $4 \%$ paraformaldehyde and treated briefly with $0.1 \mathrm{M}$ glycine in PBS (pH 7.4), followed by $0.3 \%$ Triton X-100 buffer. Cells were later stained with anti-LC3 Ab $(1: 200)$ overnight at $4^{\circ} \mathrm{C}$ and then with Alexa Fluor 488-conjugated antirabbit $\mathrm{Ab}$ $(1: 200)$ for $1 \mathrm{~h}$ at RT. TOTO-3 iodide (642/660) (Invitrogen) was used for nuclei staining. Glass slides were washed and mounted with $95 \%$ glycerol in PBS. Confocal microscopy was carried out on a Radiance 2100 laser scanning confocal microscope (Bio-Rad Laboratories) equipped with a krypton/argon laser and a red laser diode. To reduce bleedthrough, confocal images were acquired sequentially. Noise reduction was achieved by 'Kalman filtering' during acquisition.

Transmission electron microscopy analysis. Samples were fixed with $4 \%$ paraformaldehyde and $2 \%$ glutaraldehyde in $0.12 \mathrm{M}$ phosphate buffer and postfixed with $1 \% \mathrm{OsO}_{4}$ in cacodylate buffer, dehydrated in ethanol and embedded in epoxy resin. Ultrathin sections $(60 \mathrm{~nm})$, obtained with ultramicrotome Ultracut $\mathrm{E}$ Reichert-Jung (Depew, NY, USA), were doubly stained with uranyl acetate and lead citrate and examined by transmission electron microscope CM 10 Philips (FEI, Eindhoven, the Netherlands).

Evaluation of autophagy. pEGFP-LC3 plasmid and the mRFP-GFP-LC3 plasmid were used. At $24 \mathrm{~h}$ after plating, the cells were transfected with either of the plasmids by using lipofectamine 2000 or Fugene. The next day, cells were treated for 8-24h with ESOM in unbuffered medium. Cells were then fixed using $2 \%$ paraformaldehyde, and autophagy was determined by quantification of the number of cells with LC3-positive organelles, counting at least 100 cells in triplicate per condition. The presence of autophagic vacuoles expressing endogenous LC3 was also assessed.

RNA interference of autophagic genes. Small interfering RNAs (siRNA) for Atg5 and Beclin-1 were synthesized by Dharmacon RNA Technologies (Milano, Italy) (siGENOME SMART pool). For siRNA transfection, Me30966 and WM793 cells were seeded in unbuffered medium at $30 \%$ confluence in 12-well tissue dishes transfected with $5 \mathrm{nM}$ of specific siRNA by using Interferin (PolyPlusTransfection, Lafayette, CO, USA) following the manufacturer's protocol. The silencing efficacy of the various siRNAs was assessed by WB analysis of Atg5 and Beclin-1 proteins using polyclonal antibodies. At $48 \mathrm{~h}$ after transfection, ESOM $(160 \mu \mathrm{M})$ was added and cells analysed for apoptosis.

Statistical analysis. Differences between groups were analysed by the Mann-Whitney test, Student's t-test or by one-way analysis of variance as appropriate. Data in the text are expressed as mean \pm S.D. and the $P$-values reported are two-sided.

\section{Conflict of interest}

The authors declare no conflict of interest.

Acknowledgements. This work was supported by the Italian Association for Cancer Research (AIRC-ISS, grant No. 5940) and the European Commission (ChemoRes). The authors thank Mrs. Susanna Petruccelli for technical assistance.

1. Gatenby RA, Gillies RJ. A microenvironmental model of carcinogenesis. Nat Rev Cancer 2008; 8: 56-61.

2. Chiche J, Brahimi-Horn MC, Pouyssegur J. Tumor hypoxia induces a metabolic shift causing acidosis: a common feature in cancer. J Cell Mol Med 2010; 14: 771-794.

3. Tredan O, Galmarini CM, Patel K, Tannock IF. Drug resistance and the solid tumor microenvironment. J Natl Cancer Inst 2007; 99: 1441-1454.

4. Subarsky $\mathrm{P}$, Hill RP. The hypoxic tumour microenvironment and metastatic progression Clin Exp Metastasis 2003; 20: 237-250.

5. Nishi T, Forgac $M$. The vacuolar $(\mathrm{H}+)$-ATPases - nature's most versatile proton pumps. Nat Rev Mol Cell Biol 2002; 3: 94-103.

6. Swietach P, Vaughan-Jones RD, Harris AL. Regulation of tumor $\mathrm{pH}$ and the role of carbonic anhydrase 9. Cancer Metastasis Rev 2007; 26: 299-310. 
7. Cardone RA, Casavola V, Reshkin SJ. The role of disturbed $\mathrm{pH}$ dynamics and the $\mathrm{Na}+\mathrm{H}+$ exchanger in metastasis. Nat Rev Cancer 2005; 5: 786-795.

8. De Milito A, Fais S. Tumor acidity, chemoresistance and proton pump inhibitors. Future Oncol 2005; 1: 779-786.

9. Negendank W. Studies of human tumors by MRS: a review. NMR Biomed 1992; 5 303-324

10. Griffiths JR. Are cancer cells acidic? Br J Cancer 1991; 64: 425-427.

11. De Milito A, Canese R, Marino ML, Borghi M, lero M, Villa A et al. pH-dependent antitumor activity of proton pump inhibitors against human melanoma is mediated by inhibition of tumor acidity. Int J Cancer 2009; 127: 207-219.

12. Gillies RJ, Robey I, Gatenby RA. Causes and consequences of increased glucose metabolism of cancers. J Nucl Med 2008; 49(Suppl 2): 24S-42S.

13. Fais S, De Milito A, You H, Qin W. Targeting vacuolar H+-ATPases as a new strategy against cancer. Cancer Res 2007; 67: 10627-10630.

14. Gatenby RA, Gillies RJ. Why do cancers have high aerobic glycolysis? Nat Rev Cancer 2004; 4: 891-899.

15. Martinez-Zaguilan R, Seftor EA, Seftor RE, Chu YW, Gillies RJ, Hendrix MJ Acidic pH enhances the invasive behavior of human melanoma cells. Clin Exp Metastasis 1996; 14: $176-186$.

16. Martinez-Zaguilan R, Raghunand N, Lynch RM, Bellamy W, Martinez GM, Rojas B et al. $\mathrm{pH}$ and drug resistance. I. Functional expression of plasmalemmal V-type H+-ATPase in drug-resistant human breast carcinoma cell lines. Biochem Pharmacol 1999; 57: 1037-1046.

17. Luciani F, Spada M, De Milito A, Molinari A, Rivoltini L, Montinaro A et al. Effect of proton pump inhibitor pretreatment on resistance of solid tumors to cytotoxic drugs. J Natl Cancer Inst 2004; 96: 1702-1713.

18. Lu X, Qin W, Li J, Tan N, Pan D, Zhang $\mathrm{H}$ et al. The growth and metastasis of human hepatocellular carcinoma xenografts are inhibited by small interfering RNA targeting to the subunit ATP6L of proton pump. Cancer Res 2005; 65: 6843-6849.

19. Robey IF, Baggett BK, Kirkpatrick ND, Roe DJ, Dosescu J, Sloane BF et al. Bicarbonate increases tumor $\mathrm{pH}$ and inhibits spontaneous metastases. Cancer Res 2009; 69: 2260-2268

20. Sennoune SR, Luo D, Martinez-Zaguilan R. Plasmalemmal vacuolar-type H+-ATPase in cancer biology. Cell Biochem Biophys 2004; 40: 185-206.

21. Yeo M, Kim DK, Kim YB, Oh TY, Lee JE, Cho SW et al. Selective induction of apoptosis with proton pump inhibitor in gastric cancer cells. Clin Cancer Res 2004; 10: 8687-8696.

22. De Milito A, lessi E, Logozzi M, Lozupone F, Spada M, Marino ML et al. Proton pump inhibitors induce apoptosis of human B-cell tumors through a caspase-independent mechanism involving reactive oxygen species. Cancer Res 2007; 67: 5408-5417.

23. Levine B, Kroemer G. Autophagy in the pathogenesis of disease. Cell 2008; 132: 27-42.

24. Codogno P, Meijer AJ. Autophagy and signaling: their role in cell survival and cell death. Cell Death Differ 2005; 12(Suppl 2): 1509-1518.

25. Levine B. Cell biology: autophagy and cancer. Nature 2007; 446: 745-747.

26. Scherz-Shouval R, Shvets E, Fass E, Shorer H, Gil L, Elazar Z. Reactive oxygen species are essential for autophagy and specifically regulate the activity of Atg4. EMBO J 2007; 26 $1749-1760$.
27. Gonzalez-Polo RA, Boya P, Pauleau AL, Jalil A, Larochette N, Souquere S et al. The apoptosis/autophagy paradox: autophagic vacuolization before apoptotic death. J Cell Sci 2005; 118(Part 14): 3091-3102.

28. Klionsky DJ, Abeliovich H, Agostinis P, Agrawal DK, Aliev G, Askew DS et al. Guidelines for the use and interpretation of assays for monitoring autophagy in higher eukaryotes. Autophagy 2008; 4: 151-175.

29. Meijer AJ, Codogno P. Autophagy: regulation and role in disease. Crit Rev Clin Lab Sci 2009; 46: 210-240

30. Silva AS, Yunes JA, Gillies RJ, Gatenby RA. The potential role of systemic buffers in reducing intratumoral extracellular $\mathrm{pH}$ and acid-mediated invasion. Cancer Res 2009; 69: 2677-2684.

31. Huber V, De Milito A, Harguindey S, Reshkin SJ, Wahl ML, Rauch C et al. Proton dynamics in cancer. J Transl Med 2010; 8: 57.

32. Shi S, Klotz U. Proton pump inhibitors: an update of their clinical use and pharmacokinetics. Eur J Clin Pharmacol 2008; 64: 935-951.

33. Hersey $P$, Watts RN, Zhang XD, Hackett J. Metabolic approaches to treatment of melanoma. Clin Cancer Res 2009; 15: 6490-6494.

34. Huang J, Canadien V, Lam GY, Steinberg BE, Dinauer MC, Magalhaes MA et al. Activation of antibacterial autophagy by NADPH oxidases. Proc Natl Acad Sci USA 2009; 106: 6226-6231.

35. Liang XH, Jackson S, Seaman M, Brown K, Kempkes B, Hibshoosh $\mathrm{H}$ et al. Induction of autophagy and inhibition of tumorigenesis by beclin 1. Nature 1999; 402: 672-676.

36. Amaravadi RK, Yu D, Lum JJ, Bui T, Christophorou MA, Evan Gl et al. Autophagy inhibition enhances therapy-induced apoptosis in a Myc-induced model of lymphoma. J Clin Invest 2007; 117: 326-336.

37. Boya P, Gonzalez-Polo RA, Casares N, Perfettini JL, Dessen P, Larochette N et al. Inhibition of macroautophagy triggers apoptosis. Mol Cell Biol 2005; 25: 1025-1040.

38. Ostenfeld MS, Hoyer-Hansen M, Bastholm L, Fehrenbacher N, Olsen OD, GrothPedersen $\mathrm{L}$ et al. Anti-cancer agent siramesine is a lysosomotropic detergent that induces cytoprotective autophagosome accumulation. Autophagy 2008; 4: 487-499.

39. Filomeni G, Desideri E, Cardaci S, Graziani I, Piccirillo S, Rotilio G et al. Carcinoma cells activate AMP-activated protein kinase-dependent autophagy as survival response to kaempferol-mediated energetic impairment. Autophagy 2010; 6: 202-216.

40. Rossi M, Munarriz ER, Bartesaghi S, Milanese M, Dinsdale D, Guerra-Martin MA et al. Desmethylclomipramine induces the accumulation of autophagy markers by blocking autophagic flux. J Cell Sci 2009; 122(Part 18): 3330-3339.

Cell Death and Disease is an open-access journal published by Nature Publishing Group. This work is licensed under the Creative Commons Attribution-Noncommercial-No Derivative Works 3.0 Unported License. To view a copy of this license, visit http://creativecommons.org/licenses/by-nc-nd/3.0/

Supplementary Information accompanies the paper on Cell Death and Disease website (http://www.nature.com/cddis) 